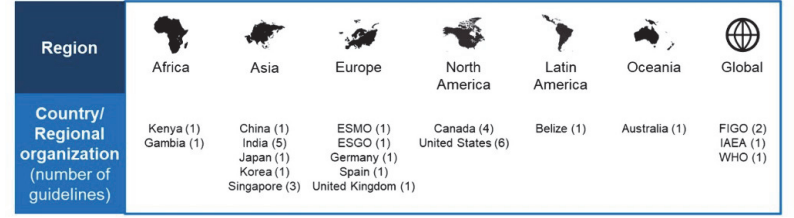

Abstract EPV069/\#40 Figure 1

geography. Included guidelines were treatment-focused and represented the latest update.

Results Thirty-four guidelines were identified (figure 1), with the majority updated 2016-2021. Seven provided only highlevel overviews of treatment modalities, and were excluded. Treatment recommendations were based on FIGO 2009 $(n=20$ guidelines), FIGO $2018(n=6)$, and TNM $(n=1)$ staging. For Stage IB2-IIA2, treatment options were diverse within/between guidelines and included radical hysterectomy $(\mathrm{RH})$, cCRT, radiotherapy. The most common recommendation was a choice of $\mathrm{RH} / \mathrm{cCRT}$ (IB2 $\mathrm{n}=12$; IIA $\mathrm{n}=18$ ), with variable treatment selection criteria between guidelines. Adjuvant cCRT/radiotherapy after $\mathrm{RH}$ was advisable with high/intermediate recurrence risk $(n=23)$. For Stage IIB-IVA, cCRT was SoC, with $\geq 67 \%$ guideline consensus. However, for Stage IIB, surgery was SoC in Japan/Germany. Ten guidelines offered Stage IVA treatment alternatives. Kenya/Gambia recommendations were distinct, offering chemotherapy alone and/or excluding cCRT. Consensus cCRT regimen was weekly cisplatin $\left(40 \mathrm{mg} / \mathrm{m}^{2}\right)$ concurrent with external beam radiotherapy followed by brachytherapy; for 6 guidelines it was unclear if cCRT included brachytherapy.

Conclusions With few exceptions, there is international consensus for cCRT as SoC for Stage IIB-IVA LACC, whereas recommendations for Stage IB2-IIA disease varied. Funding: AstraZeneca

\section{EPV070/\#401 RANDOMIZED CONTROLLED TRIAL OF THE EFFICACY OF LYMPH NODE DISSECTION ON STAGE IIICR OF CERVICAL CANCER (CQGOG0103)}

M He*, L Zhong, H Wang, Y Tang, Q Zhou, D Zou. Chongqing University Cancer Hospital, The Gynecologic Oncology Center, Chongqing, China

\subsection{6/ijgc-2021-IGCS.138}

Objectives Our goal is to assess the impact of lymph node dissection on stage IIICr of cervical cancer and to examine the specific complications of this therapy

Methods This is an national, prospective, multicenter and randomized clinical study designed to determine if patients with stage IIICr of cervical cancer have longer PFS and/or OS with lymph node dissection before Concurrent Chemoradiation therapy (CCRT) when compared to CCRT.

Results In our center, a study showed that surgical staging of women with locally advanced cervical cancer can provide more accurate information than CT/MRI scans and resulted in a longer PFS when the short diameter of image-positive lymph node $\geq 15 \mathrm{~mm} \quad(33 \mathrm{~m}$ vs. $24 \mathrm{~m}, \mathrm{p}<0.05)$. Therefore, we designed the randomized controlled trial, CQGOG0103, to determine if patients with stage IIICr of cervical cancer have longer PFS and/or OS with lymph node dissection before CCRT when compared to CCRT. Up to today, 9 patients have

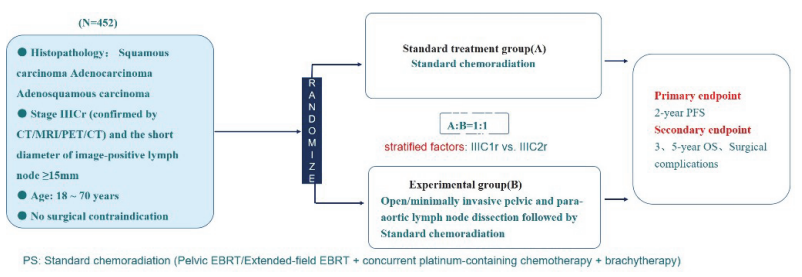

Abstract EPV070/\#401 Figure 1 Study design

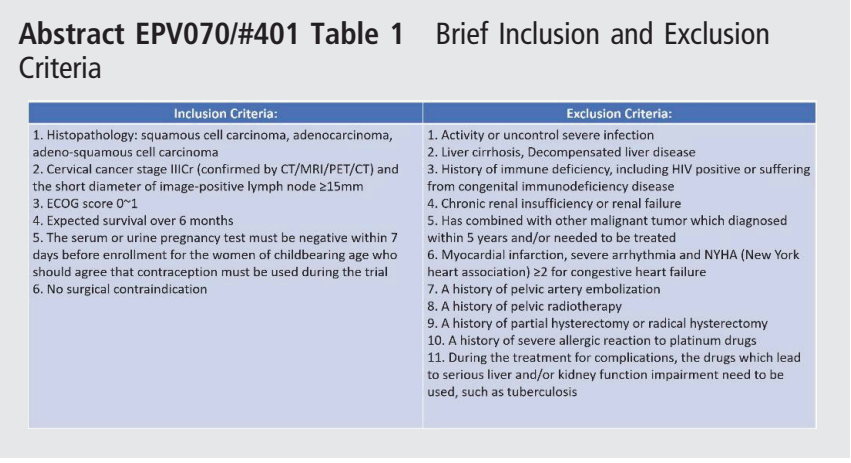

been enrolled. This trial is currently open and enrolling patients.

Conclusions Lymph node dissection may improve the prognosis of stage IIICr of cervical cancer. We will report the primary, midterm and final results about this study in the future.

\section{EPV071/\#412 DOSIMETRIC STUDY ON OCCULT UTERINE PERFORATION DURING IMAGE-GUIDANCE BRACHYTHERAPY OF CERVICAL CANCER}

${ }^{1} \mathrm{X}$ Zhao, ${ }^{2} \mathrm{H}$ Wu, ${ }^{1} \mathrm{D}$ Zou, ${ }^{1} \mathrm{Q}$ Zhou. ${ }^{1}$ Chongqing University Cancer Hospital, Gynecological Oncology Center, Chongqing, China; ${ }^{2}$ Chongqing University Cancer Hospital, Radiation Oncology Center, Chongqing, China

\subsection{6/ijgc-2021-IGCS. 139}

Objectives Based on occult perforation CT images during brachytherapy in cervical cancer, to evaluate the dosimetric parameters between $3 \mathrm{D}$ plan and $2 \mathrm{D}$ plan for providing clinical reference.

Methods The patients with cervical cancer who received intracavitary (intrauterine tandem + vaginal colpostats) were retrospectively reviewed between January 2019 to December 2020 at Chongqing University Cancer Hospital. Based on Oncentra Brachytherapy planing system, same prescription 6Gy, design $3 \mathrm{D}$ and $2 \mathrm{D}$ plan on perforated CT images respectively. Target volume, conformity index (CI), conformal index (COIN) and organs-at-risk (OARs) D2cc were assessed in two plans.

Results A total of 817 patients were included in this study. Perforations were observed in 16 patients (1.96\%). The volume of prescription dose curve in the $3 \mathrm{D}$ plan was significantly reduced $50.72 \pm 4.73 \mathrm{~cm} 3$ than $2 \mathrm{D}$ plan $(\mathrm{P}<0.05)$, but there was a similar volume of HR-CTV; the CI and COIN of $3 \mathrm{D}$ plan were promoted $0.41 \pm 0.01$ and $0.35 \pm 0.78$ than $2 \mathrm{D}$ plan $(\mathrm{P}<0.05)$, respectively; the dose received by OARs (bladder, rectum, sigmoid, small intestine) D2cc in 3D plan were significantly decerased $[(241.97 \pm 86.64)$ cGy, $(158.89 \pm 46.14)$ 


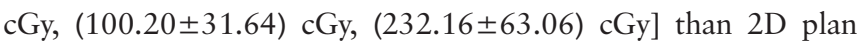
$(\mathrm{P}<0.05)$.

Conclusions Image-guidance brachytherapy of cervical cancer is helpful to detect hidden uterine perforation. When uterine perforation occurs, 3D plan can basically meet the clinical needs and is significantly better than $2 \mathrm{D}$ plan.

\section{EPV072/\#413 SUTURE GRANULOMA MIMICKING STAGE IB1 CERVICAL CANCER RECURRENCE ON VESICAL- UTERINE SPACE AFTER RADICAL TRACHELECTOMY}

${ }^{1} \mathrm{PE}$ Carvalho De Cillo*, ${ }^{1} \mathrm{H}$ Arisawa, ${ }^{2} \mathrm{O}$ Rangel, ${ }^{1} \mathrm{MGB}$ Uyeda. ${ }^{1}$ Universidade Federal de São Paulo, Gynecolgic Oncology, São Paulo - SP, Brazil; ${ }^{2}$ Universidade Federal de São Paulo, Gynecologic Oncology, São Paulo, Brazil

\subsection{6/ijgc-2021-IGCS. 140}

Objectives Introduction: Suture granuloma is a rare benign tumor caused by suture material, which usually appears several months or years after surgery. It may look identical to tumor relapse or metastatic disease on CT, PET scans and MRI, requiring histopathologic confirmation. An electronic literature search was undertaken using Medline, PubMed and Cochrane library databases for cerclage suture granuloma cases, using terms 'cerclage', 'suture granuloma', 'cervical cancer' 'metastasis' and boolean operators AND or OR, without any findings. Methods Presentation of case: 31 years-old with cervical cancer IB1 in 2014. She underwent radical trachelectomy, laparoscopic limphadenectomy and cerclage as fertility-sparing treatment. The patient attended for regular follow-up during 4 years, without signs of recurrence. 6 years after treatment, presented vaginal bleeding and fetid discharge. Physical examination: $1 \mathrm{~cm}$ exophytic bleeding lesion on anterior cervical-vaginal recess. Two biopsies were realized without any concluding findings. Pelvic MRI evidenced a cystic lobulated formation with thick walls, vascularized septs presenting wide contact with vesical trigon measuring $2.2 \times 1.5 \times 2.4 \mathrm{~cm}$, and an enlarged lymphnode in internal iliac chain of $1.3 \mathrm{~cm}$. Considering the risk of malignancy, the patient was submitted to laparoscopic resection of the lesion and lymphnode dissection.

Results The final analysis resulted in granulation tissue with no signs of malignancy in lesion or in lymphnode, thus excluding the hypothesis of malignant recurrence or metastasis.

Conclusions Discussion: Foreign body granuloma may look identical to tumor relapse or metastatic disease on image exams, thus requiring histopathological confirmation. However, the hypothesis of granulomatous reactions involving cerclage non-absorbable suture must be a differential diagnosis in clinical practice.

\section{EPV073/\#423 AN OVERVIEW OF CERVICAL CANCER EPIDEMIOLOGY AT THE ONCOLOGY GYNECOLOGY CENTER OF SANTA MARCELINA HOSPITAL- BRAZIL}

S Sanches*, M Mesquita, J Barbosa, T Almeida, M Brandão, M Silva, C Sousa, C Gomez, I Manchini. Casa de Saúde Santa Marcelina, Gynecologic Oncology, Sao Paulo, Brazil
Objectives Evaluate clinical results of patients with cervical cancer, based on statistics from the Oncology Gynecology Center of Santa Marcelina Hospital in Sao Paulo, Brazil, between 2012 to 2018 .

Methods Retrospective analysis of 370 patients' medical records, who were followed up. The data table included the following variables: age at diagnosis, symptoms, tobacco use, staging, treatments, fatal outcome, and histological type.

Results The median age of patients at diagnosis was 51 years. The major symptom was vaginal bleeding (53.65\%). Presence of smoking was reported by $33 \%$ of the patients. The histological distribution showed predominance of epidermoid carcinoma (83.6\%) over adenocarcinoma (13.8\%). About the diagnosis, $4.2 \%$ of the patients were in stage IA1, followed by IA2 2.2\%; IB1 $8.4 \%$; IB2 $6.5 \%$; IB3 1\%; IIA1 1.16\%; IIA2 2.7\%; IIB 15.4\%; IIIA 1.4\%; IIIB 41.9\%; IIIC1 1\%; IIIC2 0.84 ; IVA $5.4 \%$ and IVB $8.9 \%$. Only $20 \%$ of the patients underwent surgical treatment and the most frequent therapeutic option were radiotherapy and concomitant chemotherapy $(80 \%)$. The relapse rate was $23,24 \%$, mostly in the vagina, and about $40 \%$ of the patients had a fatal outcome in our review.

Conclusions Based on these data table, vaginal bleeding as the major symptom, the high rate of IIIB stage diagnosis, and of the fatal outcome, may be an indicative of late diagnosis of this population confirming reports from developing countries. As cervical cancer has a chance of cure if diagnosed at early stages, the results demonstrate the need for investments in educational initiatives to raise awareness among the public about the importance of cervical cancer screening.

\section{EPV074/\#427 CERVICAL CANCER: MULTICENTRIC EPIDEMIOLOGICAL STUDY}

${ }^{1} \mathrm{R}$ Arfaoui, ${ }^{2} \mathrm{MA}$ Ferjaoui ${ }^{*},{ }^{3} \mathrm{Y}$ Berrazega, ${ }^{4} \mathrm{~S}$ Khedhri, ${ }^{2} \mathrm{~K}$ Abdessamia, ${ }^{4} \mathrm{M}$ Malek, ${ }^{4} \mathrm{~K}$ Neji, ${ }^{2} \mathrm{H}$ Gmara. ${ }^{1}$ Tunis military hospital, Department of Gynecology and Obstetrics, Tunis, Tunisia; ${ }^{2}$ Tunis maternity center, Department B of Gynecologic Surgery, Tunis, Tunisia; ${ }^{3}$ Abderrahmen mami university hospital, Department Medical Oncology, Tunis, Tunisia;

${ }^{4}$ Tunis Maternity Center, Department B of Gynecologic Surgery, Tunis, Tunisia

\subsection{6/ijgc-2021-IGCS.142}

Objectives To determine the epidemiological profile of cervical cancer in Tunisia and to specify the cost of treating the disease in order to develop an effective prevention strategy.

Methods It is a retrospective descriptive, multicenter study conducted in 6 obstetrical gynecology departments over a four-year period from January 1, 2016 to December 31,2019.

Results The number of all-stage cervical cancer in the different centers was 655 cases over a four-year period; which is equivalent to 166 cases/year. The average age of our patients was 53.5 years. More than half of our population did not have health insurance, and 38\% were illiterate. The average age of sexual activity was 22.2 years. Cervical smear screening was performed in only 35 patients (17.9\% of cases). The average consultation time in the study population was 5.6 months. The most frequent reason for consultation was metrorrhagia in $63 \%$. A clear predominance of squamous cell carcinoma $(82 \%)$ was noted. Tumors were classified according to the FIGO 2009 classification: $23.5 \%$ were diagnosed at an early stage $(<\mathrm{IB} 1)$ and $76.3 \%$ at advanced stages (IB2 up to IV). 